conveniently situated in each room. The advanced laboratory is fitted with a 'service shelf' 15 in. wide around three sides of the room (having gas, water and electric services thereon), which when used in conjunction with the four strong loose tables gives considerable flexibility.

The elementary laboratories and lecture rooms contain fully equipped demonstration benches, and a certain amount of local storage is provided in all rooms. All rooms have dark blinds. Free wall space is fitted with battens for fixing and hanging apparatus, and the laboratories have beams suspended from the ceiling for the usual physical experiments. Built-in slate galvanometer shelves are provided in convenient positions, those in the lecture rooms having measuring leads and 12-volt light leads connected to the demonstration benches for demonstration work with mirror galvanometer and ground glass scale.

Large yellow glass 'boards' (in place of the more usual blackboards) have been used throughout. Experimental electrical services consist of switch plugs to all working spaces, which in the elementary laboratories can be used on the 230 volts A.C. supply or alternatively on 12 volts A.c., one pair of leads only being used (designed to avoid varying voltage drops). Voltage reduction is by 'step-down' transformer and change-over switch under the supervision of the master. Direct current supplies for the advanced laboratory and all demonstration benches at 24 volts is provided from a battery of cells and an oxide cathode rectifier, interchange of voltage being carried out by plug and socket distribution board arrangement. All the D.c. equipment is located on one wall of the advanced laboratory and is available for instructional purposes.

The first floor (biology) consists of two very large well-lighted laboratories, a common preparation room, store room, greenhouse, and a small private research room for the masters' use. Except for incubator accommodation in one room and aquaria in the other, both large laboratories are identical in fitments, one portion of each room being laid out with flat-topped tables and seats for 24 students (for theoretical work), with fully equipped demonstration bench, projection screen, and 'yellow board', the other portion fitted with low benches (for 26 students) for microscope and dissecting work. These latter benches have gas points and microscope light points fitted, and are designed to accommodate microscopes and their lamps when not in use. There are two large wash-up sinks and drainers in each laboratory, with provision for draining and storing dissecting boards, and around all walls one continuous shelf allows of the display of natural history specimens and specimens 'in embryo'. North-east top lights are provided over that part of each laboratory devoted to practical work, and dark blinds are provided throughout. Storage and preparation rooms provide accommodation graded to suit all sizes from small stain bottle racks to large diagram charts.

The old building has been considerably re-modelled internally to provide the necessary accommodation exclusively for chemistry, comprising two large elementary laboratories (one for fifth-form work), two large lecture rooms, an advanced (specialist) laboratory for 14, two balance rooms, the usual store and preparation rooms and a small science library. Existing fittings have been modernized, new chemistry working benches provided where necessary, and new ranges of fume cupboards in the fifth-form laboratory and advanced laboratory have been installed, ventilated by a centrifugal fan and acidresisting ducting. New roof lights have been added to improve the natural lighting of the rooms.

The architects for the work were Messrs. Munby and Smith, of 9 Old Square, Lincoln's Inn, London.

\title{
Effect of Inbreeding of Physiologic Races of Rust
}

$\mathrm{T}$

HE recognition of the + and - strains in rust fungi and the way in which the bi-nucleate aecial condition must be preceded by mycelial fusion of + and - strains or the introduction of pycniospore-containing nectar of the opposite strain, has led to the possibility of the study of inbred physiologic races. Johnson and Newton (Can. $J$. Res., 16 ; 1938) have made a study of the effects produced in Puccinia graminis Tritici Erikss. and Henn. Cultures have been started from a single urediospore and these have been followed through their cycle, selfing being brought about by mixing pycniospore-containing nectar of pustules of the isolated race.

It is found in several of the isolated races, when selfing has been repeated for several generations, that new features are liable to appear. Thus races which originally had the normal red uredial colour have produced types with greyish-brown, orange or white uredia-features which are of extremely rare occurrence in Nature. The proportions in which these abnormalities of uredial colour appear suggest a genetic relation in which greyish-brown and orange are dominant to white, and the normal red to the other three.

Also in such inbred races types have appeared with decreased vigour of uredia as evidenced by a tendency towards or failure of the uredial pustules to break through the host epidermis; other types have shown a weakening of pathogenicity, as compared by inoculation of named varieties of wheat, and still others have failed to produce aecia and instead formed urediospores or teliospores on the barberry.

The authors point out that mutations have been reported several times for the rusts, and the evidence from the present work suggests that certain physiologic races are in a mutable condition. Owing to the multiplicity of physiologic races and the heterothallic nature of the rusts, in Nature the mutants will seldom occur in the homozygous condition. When inbreeding is maintained for several generations, it is probable that homozygous recessive types are segregated (or possibly additional mutations occur during the selfing stages on barberry).

In the group of the rusts, short-cycle forms are of relatively common occurrence and may tend towards the production of autoecious species. In this connexion it is of interest to find in Puccinia graminis a mutant in which the aecial stage is eliminated, though the urediospores produced on the barberry are only capable of infecting wheat seedlings, so that the physiologic race is still heteroecious. 